L a distribución del bosque mesófilo de montaña en México está conformada por numerosas "islas" o fragmentos aislados de variadas formas y dimensiones. No es sorprendente, por lo tanto, que la distribución geográfica de muchas especies propias de estas comunidades tengan una distribución igualmente fragmentada, y que muchas presenten disyunciones notables. Sin embargo, Cyrilla racemiflora $\mathrm{L}$. (Cyrillaceae), especie que en México sólo se conoce de porciones muy húmedas de la Sierra Norte de Oaxaca, donde se han registrado precipitaciones anuales superiores a los 5,000 mm (Rzedowski y Palacios-Chávez, 1977), destaca por tener una distribución única entre los elementos florísticos de estos bosques. La especie está presente en un conjunto de áreas alejadas entre sí en las vertientes atlánticas de América del Norte y del Sur, y en el archipiélago antillano (figura 1). En opinión de Croizat (1952), esta distribución es de lo más inusual. Aunado a esto, algunos aspectos también poco comunes de su historia natural hacen de esta especie un taxon raro en este tipo de vegetación en el país.

Si bien desde que Linneo reconoció este taxon se han descrito más de una decena de especies de Cyrilla, la monografía de la familia realizada por Thomas (1960) establece que se trata de un género monotípico, cuya única especie es altamente polimórfica. Sin embargo, ésta no parece ser una visión definitiva, ya que existe la opinión de que $C$. racemiflora se restringe estrictamente al sureste de los Estados Unidos, y que el material oaxaqueño corresponde a $C$. antillana (R. Berizain, com. pers.). La ausencia de una publicación sobre estas ideas recientes obliga a seguir utilizando Cyrilla racemiflora como nombre válido para el material de Oaxaca.

La familia Cyrillaceae, netamente americana, está poco diversificada

\title{
Plantas leñosas raras del bosque MESÓFILO DE MONTAÑA IV. \\ Cyrilla racemiflora L. (Cyrillaceae)
}

\section{Claudia Gallardo, Jorge Meave y Armando Rincón}

Laboratorio de Ecología, Facultad de Ciencias, Universidad Nacional Autónoma de México, Coyoacán, México 04510, D.F., México.

ya que sólo incluye otros dos géneros (Purdiaea y Cliftonia). Una de las polémicas más interesantes en torno a este grupo se refiere a su origen. Croizat (1952) propuso que su línea ancestral proviene de Africa. En contraste, las similitudes de los representantes actuales de Cyrilla con polen del Cretácico Superior (Chmura, 1973; Müller, 1981), y con polen y madera fósil del Terciario Medio (Barghoorn y Spackman, 1949; Traverse, 1955; Frederiksen, 1980) señalan un origen antiguo y norteño, mientras que los caracteres supuestamente primitivos de las especies sudamericanas de Purdiaea apuntan hacia un origen austral (Thomas, 1960). Al margen de esta polémica, es claro que el grupo es muy antiguo y quizá la especie representa un elemento relictual en las comunidades húmedas de montaña de México, al igual que otros taxa tales como Oreomunnea mexicana (Rzedowski y Palacios-Chávez, 1977) y Ticodendron incognitum (Meave et al., 1996).

A lo largo de la distribución geográfica de C. racemiflora existe una gran heterogeneidad de hábitats. Las poblaciones norteñas se desarrollan en sitios pantanosos o inundables del sureste de Estados Unidos (de Virginia a Florida y sureste de Texas). Además, la especie está presente. en las sabanas de Belice, Nicaragua, Colombia, Venezuela y Guyana, así como en algunas serranías de Brasil. En contraste, en las Antillas, México y Panamá forma parte de los bosques húmedos montanos.

Los registros de esta especie para México son escasos y se apoyan en unas cuantas colectas depositadas en el Herbario Nacional de México (MEXU), y en un ejemplar en el Herbario Nacional de los Estados Unidos (US). Todos estos especímenes provienen de dos localidades ubicadas en la vertiente de barlovento de la Sierra de Juárez en Oaxaca, separadas por una distancia aproximada de $60 \mathrm{~km}$ y donde se desarrollan comunidades vegetales similares. En 1894, E.W. Nelson recolectó Cyrilla racemiflora por primera vez en el país, en los alrededores de Totontepec, en una altitud entre 1100 y $1700 \mathrm{~m}$. Durante un siglo estos ejemplares constituyeron la única evidencia de la presencia de la especie en territorio mexicano, y gracias a ellos ésta pudo ser incluida en la obra de Standley (1923) Trees and Shrubs of Mexico. En años recientes se han recolectado 
más ejemplares en la misma área de Totontepec, y adicionalmente nosotros localizamos a la especie en los bosques húmedos de montaña de los alrededores de Santa Cruz Tepetotutla, Municipio de San Felipe Usila, en la región conocida como la Chinantla.

C. racemiflora recibe numerosos nombres comunes a lo largo de su área de distribución, entre los que destacan "palo colorado" (Puerto Rico y República Dominicana) y "swamp cyrilla" y "leatherwood" en los Estados Unidos (Little y Wadsworth, 1964); no se han registrado nombres comunes para Oaxaca. La siguiente descripción, basada en individuos observados en Oaxaca y complementada con información publicada sobre material puertorriqueño, pretende ofrecer una idea general de la morfología de esta especie.

A pesar de que $C$. racemiflora suele crecer como un arbusto en riscos, cimas, pantanos o sabanas, en los bosques montanos húmedos de Oaxaca y las Antillas se desarrolla como un árbol de porte grande, al-

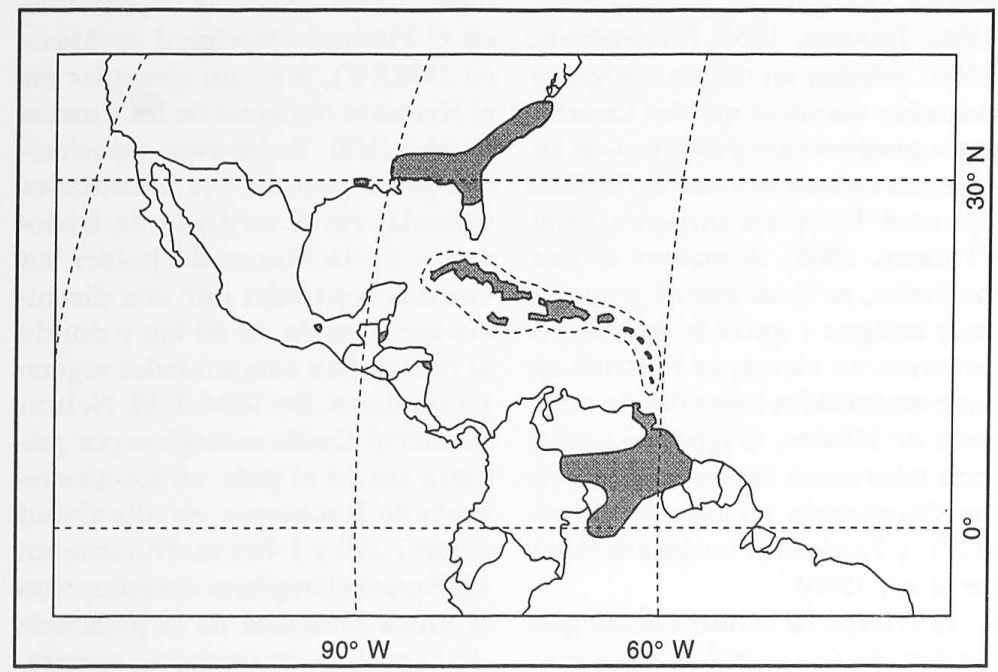

Figura 1. Distribución geográfica de Cyrilla racemiflora. Modificado de Weaver (1996). La localidad de Santa Cruz Tepetotutla se encuentra en la pequeña porción de la distribución señalada para Oaxaca.

canzando alturas cercanas a $35 \mathrm{~m}$; Weaver (1986) cita diámetros a la altura del pecho (d.a.p.) de hasta 2.6 m para esta especie en Puerto Rico. La corteza es fisurada y la madera es blanda y esponjosa hacia la base; el duramen es de color café rojizo. Los individuos grandes suelen presentar cavidades en sus troncos. En Puerto Rico, éstos constituyen los principales sitios de anidamiento de Amazona vittata, perico endémico de la isla, y de otras aves (Little y Wadsworth, 1964; Ewel y Whitmore, 1973; Weaver, 1996). En algunas regiones los individuos de C. racemiflora desarrollan nuevos tallos a partir de raíces horizontales, formando rodales clonales (Thomas, 1960), o de troncos recostados (Weaver, 1996); ninguno de estos fenómenos ha sido observado en la Chinantla. Las hojas son estrechamente elípticas y coriáceas, lustrosas en el haz y presentan un ápice agudo o emarginado; en Oaxaca miden $4-12 \mathrm{~cm}$ de largo y $1-2 \mathrm{~cm}$ de ancho. Presentan una vena media prominente en el envés, numerosas (hasta 20) venas secundarias, y una

\section{$z$
$\vdots$
0}

venación terciaria muy evidente y estrechamente reticulada. La filotaxia es alterna y las hojas tienden a aglomerarse hacia las partes apicales de las ramas. El color rojo que adquieren en la senectud permite detectar fácilmente la especie en el bosque. Al parecer en los sitios de Oaxaca los individuos no son completamente caducifolios. Las flores, diminutas y blanquecinas, se desarrollan en racimos axilares. Las flores tienen cinco (raramente seis) sépalos deltoides persistentes en la fructificación y cinco pétalos membranáceos. Los estambres (5) presentan anteras versátiles con dehiscencia longitudinal. El ovario súpero está dispuesto sobre un pequeño disco y presenta dos estigmas. Los frutos son pequeños e indehiscentes. Thomas (1960) cita informes de la ocurrencia de partenocarpia en la especie; además, menciona que existen evidencias de autoincompatibilidad, aunque ésta no ha sido probada experimentalmente. De cualquier modo, durante la época de fructificación bajo los árboles se acumulan numerosos racimos cargados de frutos.

En las localidades oaxaqueñas la especie ha sido recolectada entre los 1150 y los 1700 m s.n.m., mientras que en Puerto Rico la especie crece desde los 350 a los $900 \mathrm{~m}$ (Weaver, 1996). Esta diferencia refleja el efecto de "elevación de masas" o "Massenerhebung", propio de los sistemas insulares (Richards, 1996). C. racemiflora ha sido citada como especie dominante en comunidades antillanas, por ejemplo, en un bosque enano (elfin forest) del este de Cuba (Seifriz, 1943, citado en Howard,1968), en un bosque montano en Jamaica, donde comparte la dominancia con Podocarpus urbanii (Grubb y Tanner, 1976) y en las montañas de Luquillo, Puerto Rico (Ewel y Whitmore, 1973; Crow y Grigal, 1979; Weaver, 1986).

En la Chinantla, esta especie crece preferentemente sobre los 
filos de la sierra orientados al norte. Solamente hemos encontrado una comunidad dominada por $C$. racemiflora en un sitio conformado por amplias lomas de pendiente relativamente suave $\left(<10^{\circ}\right)$, ubicada a 1650 m s.n.m. Esta topografía contrasta con las fuertes pendientes que caracterizan a la región en general. En este sitio realizamos un muestreo en 0.1 ha utilizando el sistema de transectos propuesto por Gentry (1982). De 329 individuos incluidos en la muestra, 17 correspondieron a C. racemiflora. Esta densidad (170 ind/ha) fue casi tres veces mayor que el promedio citado por Weaver (1986) para el bosque de Luquillo (62 ind/ha). El d.a.p. promedio para los árboles de esta especie fue de $41.4 \mathrm{~cm}$, y la distribución de frecuencias de clases diamétricas mostró una escasez de individuos en las clases extremas: solamente un individuo tuvo un d.a.p. $<30 \mathrm{~cm}(13.7 \mathrm{~cm})$, y sólo uno $>60 \mathrm{~cm}(86 \mathrm{~cm})$. Weaver (1986) encontró una distribución de frecuencias semejante en un bosque bien conservado en Puerto Rico. La ausencia de individuos pequeños en ambos sitios sugiere que existen problemas de regeneración bajo un dosel cerrado.

Los individuos del bosque de Luquillo son muy longevos y de lento crecimiento (incrementos diamétricos del tronco $=0.1 \mathrm{~cm} / \mathrm{año}$; Weaver, 1986). Suponiendo que esta tasa es similar en la Chinantla, y considerando aspectos sobre el crecimiento de estos árboles mencionados por Weaver que modifican las estimaciones de edad, los árboles de nuestra región de estudio con d.a.p. de $40 \mathrm{~cm}$ y de $1 \mathrm{~m}$ tendrían edades aproximadas de 300 y 600 años, respectivamente.

Entre las 54 especies que aparecieron en el muestreo en la Chinantla, a C. racemiflora correspondió el mayor Valor de Importancia Relativa (VIR, calculado como la suma de su densidad, área basal y frecuen- cia relativas), prácticamente duplicando los valores obtenidos para Ticodendron incognitum y Pinus chiapensis, especies que le siguieron en importancia. Otras especies importantes en el sitio fueron Podocarpus matudae, Zinowiewia sp., Liquidambar styraciflua, Bejaria aestuans, Faramea schultesii y Vaccinium consanguineum. En este sitio, los árboles de Cyrilla soportan numerosas plantas epífitas, principalmente de los géneros Cavendishia, Clusia, Peperomia, Tillandsia y numerosas orquídeas.

En el límite altitudinal inferior de la distribución de Cyrilla en la Chinantla (1 $100 \mathrm{~m}$ ), la especie forma parte de la selva alta perennifolia de montaña. En dicha comunidad, las especies más importantes incluyen elementos propios de las selvas de tierras bajas (Pseudolmedia spuria, Rheedia edulis y Matayba oppositifolia, entre otras), mezclados con especies más bien propias de comunidades montanas (Clethra integerrima, Miconia trinervia, Ticodendron incognitum, Saurauia comitis-rossei y Hedyosmum mexicanum). En las porciones intermedias de su ámbito altitudinal, la especie está representada por pocos individuos que crecen en selvas bajas perennifolias sobre los filos de montaña junto con Ocotea acuminatissima, Persea aff. liebmannii y Ternstroemia sphaerocarpa.

La exploración botánica realizada en la Chinantla sugiere que $C$. racemiflora tiene una distribución muy restringida. Esto es preocupante, ya que recientemente han surgido factores que ponen en riesgo a sus poblaciones. En particular, la construcción del camino de acceso a la comunidad de Santa Cruz Tepetotutla afectó de manera importante la única porción de bosque donde sabemos que Cyrilla es abundante. $\mathrm{Al}$ margen de los individuos que ya fueron derribados durante su construcción, este camino representa un peligro potencial para la permanencia del bosque, y por lo tanto para la sobrevivencia de la especie, ya que podría acelerar la expansión de la frontera agrícola en la región. En Puerto Rico se ha observado que los disturbios ocasionados por derrumbes de tierra (Guariguata, 1990) y por huracanes y tormentas tropicales (Weaver, 1986, 1989) favorecen la regeneración de esta especie. Esto constrasta con nuestras observaciones realizadas en la Chinantla, donde $C$. racemiflora no está presente en los frecuentes derrumbes de tierra, ni en acahuales de distintas edades ubicados alrededor de los $1150 \mathrm{~m}$ (M.A. Romero, com. pers.). El posible valor ecológico de Cyrilla recemiflora en los bosques montanos húmedos de Oaxaca, así como los usos potenciales de esta especie (ornamental, medicinal, melífera, madera para construcción, etc.; Weaver, 1996), hacen de ella un objeto de estudio de gran interés y al que habría que darle prioridad.

\section{Agradecimientos}

Expresamos un agradecimiento muy especial a los pobladores de Santa Cruz Tepetotutla por el apoyo brindado durante el trabajo de campo. Lucio Lozada Pérez colaboró en la determinación de nuestros ejemplares y Marco Antonio Romero en la realización del mapa. Fernando Chiang hizo valiosos comentarios a una versión preliminar del manuscrito. Este estudio se realizó gracias al apoyo económico de la CONABIO (Proyecto P069); la visita al Herbario Nacional de los Estados Unidos fue apoyada parcialmente por el programa PADEP (UNAM).

Especimenes examinados. Oaxaca: Near Totontepec, alt 5500 to 3700 ft., E.W. Nelson \# 794, 15-28 jul. 1894 (US); Totontepec, Mpio. Totontepec, Dto. Mixe, $17^{\circ} 15^{\prime} \mathrm{N}, 96^{\circ} 00^{\prime} \mathrm{O}$, ca.1900 m, bosque mesófilo de montaña, 2 jul. 1987 (árbol de $5 \mathrm{~m}$ con flores), José Rivera \# 780 y G. Martin (MEXU); Totontepec, Mpio. Totontepec, Dto. Mixe $17^{\circ} 15^{\prime} \mathrm{N}$, 
9602' O, ca. 1900 m, bosque mesófilo de montaña, 1 jun.1990 (árbol de $35 \mathrm{~m}$ con flores), J. Rivera \# 1522 y G. Martin (MEXU); camino al río Perfume, $5.1 \mathrm{~km}$ en línea recta al SE $\left(160^{\circ}\right)$ de Santa Cruz Tepetotutla, Mpio. San Felipe Usila, Dto.

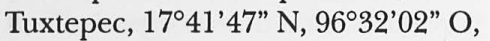
$1130 \mathrm{~m}$, selva alta perennifolia de montaña, 6 may. 1994 (árbol de 30 $\mathrm{m}$, con flores), P. Osorio \# 136 (MEXU); Monte Pan, $3.1 \mathrm{~km}$ en línea recta al SE $\left(135^{\circ}\right)$ de Santa Cruz Tepetotutla, Mpio. San Felipe Usila, Dto. Tuxtepec, $17^{\circ} 42^{\prime} 57^{\prime \prime} \mathrm{N}$, 96³2'09" O, $1050 \mathrm{~m}$, selva alta perennifolia de montaña, 13 may. 1994 (árbol de $35 \mathrm{~m}$, con flores), P. Osorio \# 158 (MEXU); $4.8 \mathrm{~km}$ en línea recta al SE $\left(164^{\circ}\right)$ de Santa Cruz Tepetotutla, Mpio. San Felipe Usila, Dto. Tuxtepec, $17^{\circ} 41^{\prime} 47^{\prime \prime} \mathrm{N}$, 96 32' 15" O, $1670 \mathrm{~m}$, bosque mesófilo de montaña, 4 jun. 1995 (árbol de $18 \mathrm{~m}$, con flores), C. Gallardo \# 1408 y A. Rincón (MEXU); 4.5 km en línea recta al SE $\left(160^{\circ}\right)$ de Santa Cruz Tepetotutla, Mpio. San Felipe Usila, Dto. Tuxtepec, $17^{\circ} 42^{\prime} 03^{\prime \prime} \mathrm{N}$, 96³2'36" O, $1640 \mathrm{~m}$, bosque mesófilo de montaña de Cyrilla racemiflora, 26 nov. 1998 (árbol de $15 \mathrm{~m}$, con frutos), Armando Rincón \# 807, C. Gallardo, J. Meave y Y. Arellano, (MEXU); $4.5 \mathrm{~km}$ en línea recta al SE $\left(160^{\circ}\right)$ de Santa Cruz Tepetotutla, Mpio. San Felipe Usila, Dto.

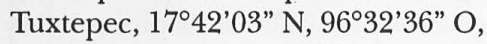
$1640 \mathrm{~m}$, bosque mesófilo de montaña de Cyrilla racemiflora, 28 nov. 1998 (árbol $25 \mathrm{~m}$, con frutos), Jorge Meave \# 2055, A. Rincón, C. Gallardo y Y. Arellano (MEXU).

\section{Literatura citada}

Barghoorn K.S. y W. Spackman Jr. 1949. A preliminary study of the flora of the Brando Lignite. American Journal of Science 247:33-39.
Chmura C.A. 1973. Upper Cretaceous (Campanian-Maastrichtian) angiosperm pollen from the western San Joaquin Valley, California, U.S.A. Palaeontographica B141:89-171.

Croizat L. 1952. Manual of phytogeography or account of plant dispersal throughout the world. Junk, La Haya.

Crow T.R. y Grigal D.F. 1979. A numerical analysis of arborescent communities in the rain forest of the Luquillo Mountains, Puerto Rico. Vegetatio 40: 135-146.

Ewel J.J. y Whitmore J.L. 1973. The ecological life zones of Puerto Rico and the U.S. Virgin Islands. Res. Pap. ITF-18. United States Department of Agriculture, Forest Service, Institute of Tropical Forestry. Río Piedras, Puerto Rico.

Frederiksen N.O. 1980. Sporomorphs from the Jackson group (Upper Eocene) and adjacent strata of Mississippi and Western Alabama. United States Geological Survey Professional Paper 1084:1-75.

Gentry A.H. 1982. Patterns of neotropical plant species diversity. En: Hecht M.K., Wallace, B. y Prance, G.T. Edrs. Evolutionary Biology. Plenum Press, Nueva York, 1-84.

Grubb P. y Tanner E.V.J. 1976. The montane forests and soils of Jamaica: a reassessment. Journal of the Arnold Arboretum 57: 313-368.

Guariguata M. R. 1990. Landslide disturbance and forest regeneration in the upper Luquillo Mountains of Puerto Rico. Journal of Ecology 78: 814-832.

Howard R.A. 1968. The ecology of an elfin forest in Puerto Rico, I. Introduction and composition studies. Journal of the Arnold Arboretum 49:381418.

Little E.L. Jr. y Wadsworth F. H. 1964. Common trees of Puerto Rico and the Virgin Islands. Agric. Handb. 249, United States Department of Agriculture. Washington, D.C.
Meave J., Gallardo C. y Rincón A. 1996. Plantas leñosas raras del bosque mesófilo de montaña II. Ticodendron incognitum Gómez-Laurito \& Gómez P. (Ticodendraceae). Boletín de la Sociedad Botánica de México 59:149152.

Müller J. 1981. Fossil pollen records of extant angiosperms. Botanical Review 47:1-142.

Richards P.W. 1996. The Tropical Rainforest. $2{ }^{\text {a }}$ ed. Cambridge University Press, Cambridge.

Rzedowski J. y Palacios-Chávez R. 1977. El bosque de Engelhardtia (Oreomunnea) mexicana en la región de la Chinantla (Oaxaca, México): una reliquia del Cenozoico. Boletín de la Sociedad Botánica de México 36:93-127.

Standley P.C. 1923. Trees and Shrubs of Mexico. Part III. Contributions of the United States National Herbarium 23:673.

Thomas J.L. 1960. A monographic study of the Cyrillaceae. Contributions from the Gray Herbarium of Harvard University 186:1-114.

Traverse A. 1955. Pollen Analysis of the Brandon Lignite of Vermont. United States Department of the Interior, Bureau of Mines, Washington D.C.

Weaver P.L. 1986. Growth and age of Cyrilla racemiflora $\mathrm{L}$. in montane forests of Puerto Rico. Interciencia 11: 221-228.

Weaver P.L. 1989. Forest changes after hurricanes in Puerto Rico's Luquillo Mountains. Interciencia 14:181192.

Weaver L.P. 1996. Cyrilla racemiflora L. Swamp cyrilla, Cyrillaceae. Cyrilla family. S.O. ITF-SM-78. United States Department of Agriculture, Forest Service, Southeastern Forest Experiment Station, International Institute of Tropical Forestry. Nueva Orleáns. 